\title{
Algunas reflexiones sobre lo que requiere un profesor para enseñar: El caso del Conocimiento Profesional del Profesor
}

Por: Elías Francisco Amórtegui ${ }^{1}$

Recibido: 28- 02-2010

Aceptado: 23-11-2010

"Las universidades eran como colegios, instituciones para preparar el más prestigioso de los profesionales, el más alto nivel escolar, el profesor"

Traducido de Shulman (1986).

\section{Resumen}

El presente ensayo pretende plantear algunas reflexiones sobre cómo se ha configurado la línea de investigación Conocimiento Profesional del Profesor, cuáles han sido sus principales propuestas, sus similitudes y diferencias, sus puntos de encuentro y desencuentro, cuál ha sido su objeto, sus metodologías, y algunas reflexiones particularmente para el caso de los profesores de Biología, entre otros aspectos. Dada la inmensidad de autores que abordan este tema, para este escrito me centraré particularmente en las propuestas de Shulman, Bromme, Grossman, Porlán y Rivero y Park y Oliver. Es de resaltar que los planteamientos son fruto de las discusiones llevadas a cabo en el seminario Conocimiento Profesional del Profesor de Ciencias, ofrecido en conjunto para los programas de Maestría en Educación (Universidad Pedagógica Nacional) y Doctorado Interinstitucional en Educación (Universidad Pedagógica Nacional, Universidad Distrital Francisco José de Caldas y Universidad del Valle).

Palabras clave: Conocimiento Profesional del Profesor, Conocimiento Profesional del Profesor de Biología, Conocimiento Didáctico del Contenido.

\section{Abstract}

This essay aims to establish some reflections about how the research line Professional Knowledge of the Teacher has been made up, what its main proposals, similarities, differences, agreement and disagreement points have been, which has been its objective, methodologies and some reflections particularly for biology teachers, among other aspects. Given the fact that there are many authors who work on this issue, for this writing I will focus particularly on Shulman, Bromme, Grossman, Porlán-Rivero and Park-Oliver proposals. It is important to remark that the approaches are based on discussions carried out in the seminar Sciences Teacher Professional Knowledge, offered as a whole for the Master's degree in Education (Universidad Pedagógica Nacional) and $\mathrm{PhD}$ in Education (Universidad Pedagógica Nacional, Universidad Distrital Francisco José de Caldas and Universidad del Valle).

1 Licenciado en Biología. Candidato a Magíster en Educación Universidad Pedagógica Nacional. Joven Investigador Colciencias. folkerpan@ hotmail.com. 
Keywords: Professional Teacher Knowledge, Biology Teacher Professional Knowledge, Pedagogical Content Knowledge.

\section{DESARROLLO}

Generalmente se le ha atribuido a Lee Shulman ser uno de los pioneros en la línea de investigación Conocimiento Profesional del Profesor. Sin embargo, es necesario realizar algunas precisiones sobre la manera en la que se ha fundado este objeto de investigación; en este sentido, es clave entender el contexto en el que se desarrolla su propuesta. El estudio de Shulman (1986) se desarrolla en el ámbito de una reforma educativa, particularmente en Estados Unidos, dado que muestra a partir de una revisión documental sobre los reportes educativos del estado de California, cómo durante el siglo XIX, existían alrededor de 20 categorías que se evaluaban a los profesores, entre las cuales se encontraban disciplinas como gramática, aritmética, geografía, historia natural entre otras, representando así el 95\% de las categorías evaluadas; la investigación de Shulman resalta que tan solo un 5\% de las disciplinas evaluadas era representado por la pedagogía.

El primer análisis sobre la situación que aborda Shulman, es que dichos aspectos mencionados mostraban el énfasis de las evaluaciones sobre el contenido que enseñaban los profesores, dejando de lado una gran cantidad de elementos que se incluyen en la actividad docente, lo cual permite hacernos cuestionamientos tales como dónde está lo pedagógico, lo didáctico y si nos refiriéramos particularmente a los profesores de Biología, dónde está lo relacionado con las salidas de campo para la enseñanza de la ecología, el trabajo sobre las ideas alternativas de los estudiantes acerca del concepto de "lo vivo" y la discusión pedagógica sobre la enseñanza de la Biología en contextos particulares.

Continuando con la discusión sobre los planteamientos de Shulman, éste plantea que cien años después, en pleno siglo $X X$, ocurre totalmente todo lo contrario en relación con los resultados hallados en su investigación. Ahora el énfasis de las evaluaciones a los maestros se ha centrado no solo en disciplinas, sino en aspectos como el manejo de los estudiantes, la organización curricular, el conocimiento sobre las políticas educativas, entre otros, los cuales son importantes, pero no son los únicos aspectos que debería saber un docente.

Bajo esta mirada podríamos preguntarnos, y para el caso de los profesores de Biología, ¿Dónde queda aquello que Valbuena (2007) ha denominado como el Conocimiento Disciplinar Biológico, su estructuración, sus principales contenidos? Y aun más importante ¿dónde queda la relación de dicho Conocimiento con el Conocimiento del Profesor?

Retomando la discusión, bajo esta panorámica, son pocos los cuestionamientos sobre lo que Shulman denomina The Subject matter content, y sobre cómo éste debe ser transformado para ser enseñado en el aula de clases, lo cual implica pensar en lo que el mismo autor ha denominado el "paradigma perdido". En estas condiciones, este autor propone resolver la pregunta ¿cuáles son los dominios y categorías del conocimiento del profesor? Propone entonces, el conocimiento del contenido, el conocimiento pedagógico del contenido y el conocimiento curricular. El primer conocimiento se refiere a la organización de aquello que 
enseña el profesor, particularmente sobre lo que Schawb (1978) citado en Shulman (1986) denomina estructura sustantiva (cuerpo teórico) y estructura sintáctica (formas de validación). Aquí surgen algunas preguntas, por ejemplo ¿por qué no llamarlo conocimiento de la disciplina? ¿Acaso es porque pueden existir maestros que no enseñan disciplinas?

Para el caso de profesores de Biología, dicha estructura sustantiva correspondería según Sánchez (2007) y Valbuena (2007) a la organización de los contenidos del Conocimiento Biológico (lo cual podría ser desde una visión sumativa de conceptos y ramas como fisiología, histología o desde una visión mucho más sistémica, teniendo en cuenta conceptos estructurantes y as pectos histórico-epistemológicos); siguiendo con la estructura sintáctica, ésta corresponde según Sánchez (2007) y Valbuena (2007) a las formas de producción del Conocimiento Biológico (en donde se tienen en cuenta aspectos como la observación, las narraciones históricas y la experimentación).

El segundo conocimiento se refiere al conocimiento para la enseñanza, el cual incluye los tópicos generales de enseñanza, las analogías, las ilustraciones, en general sobre el aprendizaje y enseñanza de los estudiantes. Por último, el tercer conocimiento hace referencia a los materiales de instrucción, y a los programas de enseñanza.

Se podrían hacer algunas reflexiones aquí sobre la propuesta de Shulman, aunque se reconoce que es un primer paso para configurar la línea de investigación sobre el Conocimiento del Profesor. Su propuesta se queda corta en términos de reconocer las relaciones que existen entre los tipos de conocimientos, tampoco explicita la importancia del contexto en la configuración de la enseñanza y cuál de esos componentes es el integrador de ese conocimiento del docente.

Aquí se debe reconocer sin embargo que la propuesta de Shulman corresponde a una revisión bibliográfica y no a una investigación propiamente dicha que haya contado con la sistematización de experiencias de sujetos investigados, de allí la propuesta que hará en 1987. Antes de entrar en dicha discusión, existen otros dos aspectos claves que Shulman reconoce en su primera propuesta: las fuentes y el carácter profesional del Conocimiento del Profesor.

Frente a las fuentes propone la disciplina empírica, relacionada con los principios que resultan de las investigaciones empíricas, lo cual considero correspondería a lo que autores como Porlán y Rivero (1998) denominan como fuente académica; propone además la experiencia práctica como aquella que guía los principios teóricos, lo cual corresponde a otros autores como la fuente experiencial, y por último Shulman propone el razonamiento ético o moral, enmarcados en las normas y valores que deberían tener los profesores, a esto Porlán y Rivero (1998) y Carr (1993) le dan un peso muy fuerte, pues para el primero corresponde a un saber metadisciplinar ontológico del profesor, y para el segundo, la perspectiva ideológica del maestro que orienta su enseñanza.

El aspecto de lo profesional mantiene un poco la idea del título de este ensayo, aunque Shulman enmarca su propuesta en el Conocimiento del profesor, cabría preguntarse ¿por qué no lo enmarca en el conocimiento profesional del profesor? Este no es un tema al parecer clave en su investigación, aunque plantea que lo profesional se refiere a que el maestro no solo reproduce conocimiento, también lo produce, y es capaz de comunicar las razones por las que actúa. Se 
queda corto en la discusión sobre lo profesional, que luego será fuertemente retomado en las discusiones de autores como Carr (1993) y Contreras (1997), desde una perspectiva sociológica, con planteamientos estructuralistas, funcionalistas y marxistas.

Por ultimo, dado que la propuesta de Shulman es más teórica que a partir de una investigación, sugiere tres formas posibles en las que se organizaría el Conocimiento del Profesor: el conocimiento proposicional, el conocimiento de caso y el conocimiento estratégico. Sin embargo, de nuevo la profundidad sobre este aspecto es muy corta y ni siquiera es retomada en sus publicaciones posteriores.

En términos generales, Shulman otorga los cimientos de la línea de investigación Conocimiento del Profesor, que luego será transformada en Conocimiento Profesional del Profesor. Se debe reconocer además que su principal propuesta es la del Pedagogical Content Knowledge (PCK) que se discutirá más adelante. Es posible inclusive, encontrar trabajos anteriores a los de Shulman, tal es el caso de Elbaz (1983) y Leinhardt y Smith, (1985), sin embargo, tan solo los del primer autor han sido considerados como estructurantes en esta línea de investigación.

Antes de pasar a la siguiente propuesta, quisiera retomar el encabezado "He who can, does. He who cannot, teaches" el cual aplica perfectamente para nuestro ámbito colombiano, pues luego de 23 años de la publicación de Shulman, el que no puede, enseña, basta con mirar las convocatorias para el denominado "concurso" docente.

Dejando de lado ese panorama un poco desalentador, existe un cambio grandísimo entre la propuesta de Shulman de 1986 y la de 1987. Aunque ambas se enmarcan en reformas educativas, en la segunda, amplía los componentes del Conocimiento del Profesor, reconfigura las fuentes de dicho conocimiento y las formas como se organiza en la práctica.

En cuanto a los componentes, considera siete: conocimiento del contenido, conocimiento didáctico general, conocimiento del currículo, conocimiento pedagógico del contenido, conocimiento de los alumnos y de sus características, conocimiento de los contextos educativos y conocimiento de los objetivos, las finalidades y los valores educativos y de sus fundamentos filosóficos e históricos.

Aunque en esta propuesta retoma muchos más componentes, las relaciones entre estos siguen siendo difusas, pues considera que las relaciones son de amalgama o de mezcla entre una materia y otra, sin embargo dicha relación es lo fundamental desde otras perspectivas que corresponden al pensamiento sistémico y complejo, tal como lo retoma Valbuena (2007), Perafán (2004) y Park y Oliver (2008). Un gran aporte en esta propuesta de Shulman (1987) es que a diferencia del artículo de 1986, en donde no diferenciaba el estatus o la importancia de los tres componentes que citaba, en éste explicita la importancia que tiene el PCK, pues es el que identifica los cuerpos de conocimientos distintivos para la enseñanza y es además el que permite distinguir entre la comprensión del especialista en un área del saber y la comprensión del pedagogo; este conocimiento es el exclusivo del maestro, es su propia forma especial de comprensión profesional.

Algunos autores como Gess Newsome (1999) reconocen que el aporte fundamental de Shulman fue configurar el PCK como aquel cuerpo de conocimientos para la enseñanza. Con relación a 
las fuentes no existe un cambio considerable con relación a su propuesta del año anterior, reconoce cuatro, la formación académica, los materiales y el contexto, la investigación sobre la escolarización, y la experiencia.

En cuanto a la organización en la práctica, propone seis acciones: comprensión, transformación, enseñanza, evaluación, reflexión, nuevas maneras de comprender. Estas acciones corresponden a lo que el docente realiza en su práctica cotidiana, sin embargo, algunas de estas acciones son retomadas por otros autores como Valbuena (2007) y Park y Oliver (2008) como componentes del Conocimiento Didáctico del Contenido (CDC) para el primer autor, y PCK para el segundo, tal es el caso de la evaluación.

Quisiera llamar la atención aquí sobre un aspecto que se resalta de manera incipiente, sobre todo en la propuesta de Shulman de 1986. Actualmente se ha apostado en la formación de profesores al modelo del profesor investigador reflexivo, fundamentado principalmente desde las propuestas de Porlán (1997), Schön (1987), e Imbernón (1997, 1998) y para el caso particular de profesores de Biología, Valbuena (2007). Sin embargo estos autores reconocen muy poco en las propuestas de Shulman un primer interés por aquello de los procesos de reflexión en el Conocimiento del Profesor. Al respecto Shulman (1986) plantea que es la metacognición y la reflexión sobre sí mismo lo que distingue al maestro del arquitecto $\mathrm{u}$ otro profesional. Posteriormente en 1987, coloca la reflexión como una acción pedagógica sobre el desempeño del profesor. Claro está que sobre la reflexión existen muchos aspectos por discutir, pero se debe reconocer que Shulman hace un intento, un poco incipiente, pero novedoso para la época y contexto de su propuesta, en relacionar la reflexión-metacognición con el Conocimiento del Profesor.

Bajo este panorama, espero se haya suscitado en el lector la siguiente pregunta, ¿a qué se debe el cambio de tres a siete componentes del Conocimiento del Profesor en las propuestas de Shulman de 1986 y 1987? ¿Por qué le da un mayor estatus al PCK en 1987 y no en 1986? Es importante contrastar ambas propuestas. La primera correspondió a una revisión teórica y a una postulación hipotética como la denomina Gess-Newsome (1999); a diferencia de esto, la segunda propuesta correspondió a una investigación propiamente dicha realizada con profesores nóveles y experimentados de literatura.

Está claro entonces que la investigación sobre la práctica docente de un maestro, es decir sobre su quehacer cotidiano permitió la reelaboración de dicha propuesta, lo cual reafirma el planteamiento de Porlán y Rivero (1998) retomada por muchos investigadores posteriormente, de que el Conocimiento del Profesor se construye en su práctica, en su puesta en escena como docente. Reafirma además la idea de que la sistematización de esta práctica del maestro es lo que permite construir conocimiento sobre lo que requiere y de dónde bebe el maestro para enseñar.

Posterior a las propuestas que se han desarrollado hasta el momento, Grossman (1990), al igual que Shulman en el contexto estadounidense, desarrolla una investigación con profesores de ingles, en donde reconoce cuatro áreas generales del conocimiento del profesor: conocimiento pedagógico general, conocimiento de la materia, conocimiento del contexto y conocimiento pedagógico del contenido. 
Hasta este punto no existe nada novedoso. Lo Nuevo está en considerar este último conocimiento como el integrador de los demás conocimientos y sobre el cual giran los otros tres componentes. A diferencia de las propuestas de Shulman, Grossman hace énfasis en las relaciones. El PCK se relaciona de manera bidireccional con el conocimiento de la materia, el conocimiento pedagógico general y el conocimiento del contexto, sin embargo no existe ninguna relación entre el conocimiento del contexto y el conocimiento pedagógico general, o entre éste y el conocimiento de la materia. Las relaciones únicamente se representan por flechas, pero no se explicita el tipo de relación (conlleva, implica, afirma, obedece a, etc.), lo cual sí se explicita en la propuesta de Park y Oliver (2008).

A diferencia de Shulman, Grossman pone un gran valor al contexto y los intereses de los alumnos para la definición y estructuración de los contenidos curriculares y de las estrategias de enseñanza en el marco del conocimiento curricular. Sin embargo, ninguno de estos dos autores ha tenido en cuenta aspectos como las concepciones de los profesores y su importancia en la estructuración del Conocimiento Profesional.

Con relación al carácter Profesional del conocimiento del profesor, Grossman al igual que Shulman, toca este aspecto tan solo tangencialmente. Grossman plantea que esas cuatro áreas del conocimiento del profesor pueden ser vistas como las esquinas del trabajo emergente sobre el conocimiento profesional del profesor para la enseñanza, sin embargo no desarrolla más esta idea.

Durante estas cinco páginas he hecho referencia a la línea de investigación Conocimiento del Profesor, particularmente en el ámbito anglosajón. Antes de introducir los planteamientos de Porlán y Rivero, me parece pertinente retomar los planteamientos de otro autor que corresponde a otro contexto, para este caso, el contexto Alemán.

Bromme (1988) realiza una revisión bibliográfica sobre los estudios sobre el conocimiento de los profesores. Su trabajo se titula "Conocimientos profesionales de los profesores", solo desde este título se desprenden inquietudes tales como ¿existen varios conocimientos profesionales del profesor? ¿Ya no es uno como planteaba Shulman y Grossman?

Bromme propone como conocimientos del profesor: conocimiento de la disciplina, conocimiento curricular, conocimiento sobre la clase, conocimiento sobre lo que los alumnos aprenden, metaconocimientos, didáctica de la asignatura, conocimiento pedagógico. En términos generales, la propuesta de este autor podría resultar un poco ambigua; por una parte no hay ninguna relación entre estos conocimientos, lo que significa que son totalmente independientes unos de otros, por otra parte no se explicita cuál es el estatus de esos conocimientos, todos estarían al mismo nivel, ninguno sería más estructurante que otro, ni más ni menos integrador que otro.

Por otra parte, el conocimiento sobre la clase es demasiado amplio ¿a qué se refiere particularmente? Este conocimiento desde mi perspectiva podría corresponder al conocimiento contextual, el cual en algunos casos puede ser integrador (Park y Oliver, 2008). La discusión sobre lo "profesional" del profesor parece quedar un poco corto en su discusión; implícitamente se podría relacionar con la intención de poner la labor docente al mismo nivel de otras labores 
como la de arquitecto y médico, pero en su trabajo no muestra un amplio desarrollo sobre este tema.

Cabe resaltar que en algunos casos se maneja indistintamente saber y conocimiento, pues se refiere a lo que conoce el profesor como conocimiento profesional y como saber profesional. Considero desde mi revisión, que saber y conocimiento son dos estatus epistemológicamente muy diferentes, por lo que manejarlos indistintamente y sin la debida reflexión sobre ello puede ser un obstáculo en la elaboración teórica de esta línea de investigación. Para esta discusión el lector podría revisar los trabajos de Vargas (2006) y Moreno, Rodríguez, Torres, Mendoza y Vélez (2008).

Bromme arroja dos grandes aportes, el primero tiene que ver con que es en la práctica, o la "sabiduría de la praxis" en donde se construyen los conocimientos profesionales del profesor, sin embargo pareciera que su idea implica que a mayor años de experiencia mayores conocimientos profesionales, lo cual considero puede ser un poco reduccionista, pues existen otros aspectos que favorecen la construcción del conocimiento del profesor, por ejemplo la reflexión y la investigación (Valbuena, 2007). El segundo aspecto clave de su propuesta son los metaconocimientos, los cuales corresponden a la perspectiva filosófica del profesor. Sin embargo, se pierde riqueza en su propuesta pues estarían al mismo nivel que los otros conocimientos, por tanto ¿qué tienen de metaconocimientos si están al mismo nivel? Desde mi perspectiva y como se verá más adelante con Porlán y Rivero, estos corresponden a elementos más transversales.

La propuesta de Porlán y Rivero (1998) es una de las más completas y más complejas con relación al conocimiento del profesor. En relación con las fuentes plantea la experiencia y la formación académica, las cuáles se encuentran también en las propuestas de Shulman (1986, 1987). Lo novedoso de esta propuesta es reconocer que además de los componentes que corresponden a conocimientos, existen los principios-creencias, las teorías implícitas y las rutinas y guiones. Sin embargo, podría plantearse que bajo esta propuesta, al igual que en Bromme (1988) se manejan indistintamente los conocimientos y los saberes, pues en algunas ocasiones se habla de uno y en otras ocasiones se habla del otro.

Otro aspecto fundamental de esta propuesta es que consideran elementos que tienen un estatus más transversal, los saberes metadisciplinares; cabría preguntarse entonces ¿los metaconocimientos de Bromme son equivalentes a los saberes metadisciplinares de Porlán y Rivero? Bajo una revisión detallada podría decirse que la propuesta de estos últimos es mucho más elaborada, pues les da un estatus particular en términos de que corresponden no a conocimientos o saberes, sino a ideología, epistemología y ontología, que tienen gran incidencia en la forma como se organiza dicho Conocimiento Profesional. Se podría resumir esta propuesta bajo la relación teoría-práctica, pues es en la práctica en donde se construye, y es en ésta en donde el conocimiento transita desde una perspectiva menos compleja a una perspectiva mucho más compleja, en términos de la relación de los diversos componentes.

Dado el peso y la importancia que dan estos autores a los principios y creencias, a las teorías implícitas y las rutinas y guiones del docente, podría hacerse las siguientes preguntas: ¿el conocimiento profesional del profesor es de carácter personal? ¿corresponde a una propuesta teórica para los profesores en general? ¿Existen conocimientos profesionales así como 
profesores en el contexto educativo? Es un cuestionamiento bastante complejo, en el cual Park y Oliver (2008) parecen inclinarse hacia la idea de que ese conocimiento es bastante personal, inclusive a partir de su investigación con profesores de química, plantean la idiosincrasia como un elemento constructor del PCK, pues en la práctica aunque los profesores tienen el mismo conocimiento, es personal en tanto las orientaciones que da el maestro sobre la enseñanza de las ciencias, las características de los estudiantes a los cuales enseña, las experiencias de enseñanza que ha tenido en su experiencias y las características personales del profesor, son diferentes en cada profesor.

Quisiera resaltar de la propuesta de Park y Oliver, que a diferencia de las propuestas que se han mostrado aquí, ellos explicitan en su modelo del PCK la reflexión sobre y en la acción del docente, que incipientemente había planteado Shulman (1986,1987). En términos de las relaciones, estos autores al igual que Valbuena (2007) apuestan más por la relación unidireccional o bidireccional de los diversos componentes, que por la integración de estos. Sin embargo, la propuesta se queda corta en plantear las fuentes de esos componentes del PCK.

Como se ha dado cuenta el lector, en todos los casos me he referido al PCK como aquel conocimiento que permite la transformación del conocimiento del contenido a una forma más enseñable. He optado por ser fiel a las propuestas de los autores, que en todos los casos, a diferencia de Porlán y Rivero, corresponden al contexto anglosajón, en donde se considera que aquella disciplina que se encarga de la enseñanza de los contenidos corresponde a la Pedagogía, mientras que la didáctica se remite exclusivamente a lo instrumental, o en ingles, a lo instructional. Sin embargo, en el contexto Francés y español, y por ende latinoamericano, se ha optado por traducir el Pedagogical Content Knowledge como Conocimiento Didáctico del Contenido (CDC).

Se le atribuye a Marcelo (1992) ser el primero en hablar sobre el CDC, sin embargo si se hace una revisión sobre su trabajo en el marco del "Congreso de didácticas específicas" dicho autor no explicita el por qué de esa traducción; sin embargo cabe la aclaración que dicho concepto fue introducido en algunas universidades españolas como las Universidades de Sevilla, Granada y Extremadura como una adaptación del PCK, haciendo referencia a las didácticas específicas en el contexto español. Desde mi punto de vista, esta traducción está relacionada con el objeto de estudio de la Didáctica, la cual se ha configurado como una disciplina científica autónoma (Gil, Carrascosa y Martínez, 2000), cuyo objeto corresponde más a la enseñanza y por ende a la transformación del conocimiento que hace el docente para enseñarlo en un contexto particular. ¿Si para nosotros lo pedagógico del PCK es más lo didáctico, entonces lo pedagógico para nosotros qué sería? Claramente nuestra "pedagogía" no corresponde a la "pedagogía" anglosajona, pues en nuestro contexto se refiere más una reflexión sobre el quehacer del docente, inclusive podría tener un estatus epistemológico diferente al de didáctica; de nuevo es pertinente revisar los estudios de Vargas (2006) y Moreno, Rodríguez, Torres, Mendoza y Vélez (2008).

Las discusiones sobre estos son pocas, lo que sí está claro es la trascendencia que tiene el CDC en el conocimiento del profesor, basta con ver la gran cantidad de trabajos sobre dicho conocimiento presentados en el VIII Congreso Internacional sobre Investigación en la Didáctica de las Ciencias llevado a cabo en el año 2009 en Barcelona. 


\section{CONSIDERACIONES FINALES}

Luego de estas reflexiones espero que se haya comprendido mi intención en el planteamiento del título de este ensayo, no como Conocimiento Profesional, o Conocimientos Profesionales, pues he mostrado cómo han emergido diversas propuestas en diferentes contextos sobre este tema, pero bajo el mismo objeto de investigación, aquello que requiere el profesor para enseñar. La discusión sobre esto nos lleva a reconocer cómo se ha configurado esta línea de investigación, bajo qué contextos han emergido algunas de sus propuestas, cuáles han sido los principales planteamientos, los puntos en común, los de distanciamiento y los de cuestionamientos. Claro está que quedan muchos aspectos por abordar, espero sean objeto de unas futuras disertaciones.

Termino este ensayo retomando una frase de Shulman, que algunos consideran como idealista, pero que personalmente considero resalta las implicaciones epistemológicas de ser maestro, "Those who can, do. Those who understand, teach".

\section{BIBLIOGRAFÍA:}

BROMME, R. (1988). Conocimientos profesionales de los profesores. Enseñanza de las Ciencias, 6 (1), 19-29.

CARR, W. (1989). Calidad de la Enseñanza e Investigación-Acción. Traducción de Ángel Martínez Geldhoff. Sevilla: Díada.

CONTRERAS, J (1997). La autonomía del profesorado. Madrid: Morata.

ELBAZ, F. (1983). Teacher thinking: A study of practical knowledge. London: Croom Helm.

GESS-NEWSOME, J. (1999). Pedagogical Content Knowledge: An introduction and orientation. En: GESS-NEWSOME, J. and LEDERMAN, N. (Eds.). Examining Pedagogical Content Knowledge. The Construct and its Implications for Science Education. Dordrecht, Boston, London: Kluwer Academic Publishers. pp. 3-17.

GIL-PÉREZ, D.; CARRASCOSA, J.; y MARTÍNEZ, F. (2000). La Didáctica de las Ciencias. Una disciplina emergente y un campo específico de investigación. En: Perales, F., Cañal, P. Didáctica de las Ciencias Experimentales. Teoría y práctica de la Enseñanza de las Ciencias. Alcoy: Marfil, p.p. 11-34.

GROSSMAN, P. (1990). The Making of a Teacher. Teacher Knowledge and Teacher Education. New York: Teachers College, Columbia University.

IMBERNÓN, F. (1997). La formación del profesorado. Buenos Aires: Paidos. 
IMBERNÓN, F. (1998). La formación y el desarrollo profesional del profesorado. Cuarta edición. Barcelona: Graó.

LEINHARDT, G., \& SMITH, D. (1985). Expertise in mathematics instruction: Subject matter knowledge. Journal of Educational Psychology, 77(3), 247-271.

MARCELO, C. (1992). Cómo conocen los profesores la materia que enseñan. Algunas contribuciones de la investigación sobre conocimiento didáctico del contenido. En: MONTERO, L. y VEZ, E. (Eds). Las didácticas específicas en la formación del profesorado, Santiago de Compostela; Tórculo. pp. 151-185.

MORENO, N; RODRÍGUEZ, A; TORRES, J; MENDOZA, N; VÉLEZ, L (2008) Tras las huellas del saber pedagógico Bogotá: Universidad Pedagógica Nacional.

PARK, S. y OLIVER, S. (2008). Revisiting the conceptualisation of Pedagogical Content Knowledge (PCK). Research in Science Education. 38: 261-284.

PERAFÁN, A. (2004). La epistemología del profesor sobre su propio conocimiento profesional. Bogotá: Universidad Pedagógica Nacional.

PORLÁN, R. (1996). Constructivismo y escuela. Sevilla: Díada.

PORLÁN, R. y RIVERO, A. (1998). El conocimiento de los profesores: una propuesta formativa en el área de ciencias. Sevilla: Díada.

SÁNCHEZ, P (2007). Formulación de proposiciones para el estudio de las concepciones sobre el conocimiento biológico en el marco del conocimiento profesional del profesor de biología. Tesis de Pregrado para optar al título de Licenciado en Biología. Universidad Pedagógica Nacional, Bogotá DC, Colombia.

SHÖN, D (1987). La formación de profesionales reflexivos. Madrid: Paidos.

SHULMAN, L (1987). Knowledge and Teaching. Foundations of the New Reform. Harvard Educational Review. 57 (1), 1-21.

SHULMAN, L. (2001) Conocimiento y enseñanza. Ensayo. Estudios públicos. pp. 83-196.

SHULMAN, L. (1986). Those who understand: Knowledge growth in teaching. Educational Researcher, 15 (2), 4-14.

VAlBUENA, E. (2007). El Conocimiento Didáctico del Contenido Biológico. Estudio de las concepciones disciplinares y didácticas de futuros docentes de la Universidad Pedagógica Nacional (Colombia). Tesis doctoral. Universidad Complutense de Madrid

VARGAS, G. (2006). Filosofía, Pedagogía, Tecnología. Investigaciones de Epistemología de la Pedagogía y Filosofía de la Educación. Bogotá: Universidad Pedagógica Nacional. 